\title{
Schamberg disease: uncommon reaction to a common drug
}

\section{Soumya Chatterjee MD MS}

A 75-year-old woman with long-standing rheumatoid arthritis presented with a mildly pruritic, symmetrical erythematous rash on her legs (Figure 1). She was not taking any specific therapy for her rheumatoid arthritis but had taken acetaminophen for 3 days because of knee pain. She noticed the rash after taking about 10 tablets. It began on her lower legs, spread proximally and became confluent. The patient was afebrile and had diffuse petechiae and red macules coalescing over her feet and legs (Figure 1). The rash was not palpable or warm and did not blanch on diascopy. Her erythrocyte sedimentation rate and C-reactive protein level were elevated.

Skin biopsy showed a superficial perivascular and interstitial lymphocytic infiltrate associated with extravasated erythrocytes in the papillary dermis (see Appendix 1, available at www.cmaj .ca/cgi/content/full/cmaj.081929/DC2). There was no hemosiderin deposition or karyorrhexis. Immunofluorescence was negative for perivascular or dermo-epidermal immunoglobulin or complement deposition. The pathological findings were consistent with pigmentary purpura (Schamberg disease). Other diagnostic possibilities included cellulitis, immune thrombocytopenic purpura, hypersensitivity (leukocytoclastic) vasculitis, Waldenström hyperglobulinemic purpura and cryoglobulinemic vasculitis. However, the absence of fever, local pain or warmth, thrombocytopenia, hypergammaglobulinemia, and histologic evidence of leukocytoclastic or cryoglobulinemic vasculitis ruled out these possibilities.

Schamberg disease was first described in 1901. The rash, which corresponds to extravasation of erythrocytes, usually appears on the lower legs but may spread to involve the thighs, abdomen and occasionally the arms. Although the exact pathogenesis is unknown, the capillary damage and consequent erythrocyte extravasation appear to be the result of a localized cell-mediated immune injury induced by a skinspecific subset of helper T lymphocytes. ${ }^{1}$ Histology shows

Soumya Chatterjee is with the Cleveland Clinic Lerner College of Medicine Case Western Reserve University, and the Department of Rheumatic and Immunologic Diseases, Cleveland Clinic, Cleveland, USA

Cite as CMAJ 2009. DOI:10.1503/cmaj.081929 superficial perivascular infiltration of lymphocytes, and rarely macrophages, associated with endothelial swelling and luminal narrowing, variable degrees of hemosiderin deposition, but no evidence of vasculitis. ${ }^{1,2}$

Drugs are important precipitating factors for Schamberg disease, with acetaminophen the most commonly implicated drug. ${ }^{2,3}$ Unlike the idiopathic form of Schamberg disease, most drug-induced cases resolve quickly after the offending drug is stopped. ${ }^{3}$ In our patient, the eruption gradually cleared over several weeks after the acetaminophen was stopped.

This article has been peer reviewed.

Competing interests: None declared.

\section{REFERENCES}

1. Smoller BR, Kamel OW. Pigmented purpuric eruptions: immunopathologic studies supportive of a common immunophenotype. J Cutan Pathol 1991;18:423-7.

2. Sardana K, Sarkar R, Sehgal VN. Pigmented purpuric dermatoses: an overview. Int I Dermatol 2004:43:482-8.

3. Schroeder-Devere T. Pigmented purpuric dermatoses. In: Wolff K, Goldsmith LA, Katz SI, et al., editors. Fitzpatrick's dermatology in general medicine. 7th ed. New York (NY): McGraw-Hill; 2008. p. 1633-7. 